\title{
The Mass-Function of Low Mass Halo Stars: Limits on Baryonic Halo Dark Matter
}

\author{
David S. Graff ${ }^{1}$ and Katherine Freese ${ }^{2}$ \\ University of Michigan, The Harrison M. Randall Laboratory of Physics, Ann Arbor, MI 48109-1120 USA
}

Received 9 February, 1996; accepted

submitted to ApJ Letters

\footnotetext{
${ }^{1}$ graff@umich.edu

${ }^{2}$ freese@mich1.physics.1sa.umich.edu
} 


\begin{abstract}
We derive mass functions (MF) for halo red dwarfs (the faintest hydrogen burning stars) and then extrapolate to place limits on the total mass of halo brown dwarfs (stars not quite massive enough to burn hydrogen). The mass functions are obtained from the luminosity function of a sample of 114 local halo stars in the USNO parallax survey (Dahn et al. 1995). We use stellar models of Alexander et al. (1996) and make varying assumptions about metallicity and about possible unresolved binaries in the sample. We find that the MF for halo red dwarfs cannot rise more quickly than $1 / m^{2}$ as one approaches the hydrogen burning limit. Using recent results from star formation theory, we extrapolate the MF into the brown-dwarf regime. We see that likely extrapolations imply that the total mass of brown dwarfs in the halo is less than $\sim 3 \%$ of the local mass density of the halo ( $\sim 0.3 \%$ for the more realistic models we consider). Our limits apply to brown dwarfs in the halo that come from the same stellar population as the red dwarfs.
\end{abstract}

dark matter - Galaxy: stellar content - Galaxy: halo - stars: low mass, brown dwarfs - stars: Population II 


\section{Introduction}

In this paper we determine a mass function for low mass stars and then extrapolate to derive an upper limit on the number of brown dwarfs. A key quantity for describing a stellar population is the mass function $\xi(m)$, the density of stars with mass between $m$ and $m+d m$; for $\xi(m)$ we use units \#stars $/ \mathrm{pc}^{3} / M_{\odot}$. The mass function (hereafter, MF) of halo stars can address the question of what makes up the dark matter of our Galaxy. Although it has been clear for some time that main sequence stars heavier than our sun are not sufficiently abundant to explain the mass of the Galaxy, the question has remained whether or not there can be large numbers of lower mass objects. Very low mass stars or substellar objects have been considered the most plausible candidates for baryonic dark matter. [White dwarfs, another possibility for baryonic dark matter, are not considered in this paper.] Recent work has ruled out red dwarfs, stars just barely massive to burn hydrogen $\left(m>0.092 M_{\odot}\right)$, as a significant source of dark matter (Graff \& Freese 1996; Boeshaar, Tyson \& Bernstein 1994; Bahcall et al. 1994). Brown dwarfs, star-like objects that are just barely too small to burn hydrogen, are the remaining low mass candidates. These are extremely difficult to observe optically (see e.g., Burrows and Liebert 1993 for a review) although there have been searches for them with gravitational microlensing (Alcock et al. 1995, Ansari et al. 1996).

As our main result, we determine a MF of halo red dwarfs, and then extrapolate into the brown dwarf regime to obtain an upper limit on the number of halo brown dwarfs. If brown dwarfs are a primary component of halo dark matter, then we would expect a steeply rising MF as we go to decreasing mass. Instead, we show that likely extrapolations imply that brown dwarfs are not nearly numerous enough to be a significant component of halo dark matter. As a caveat, let us point out that our limits apply only to brown dwarfs in the halo that come from the same stellar population as the red dwarfs (see the discussion at the end of the paper). We also rely on recent results from star formation. The goal of our paper is to show that brown dwarfs coming from known stellar populations are not present in great abundance. 1

Unfortunately, the MF cannot easily be obtained by observations, since it is difficult to measure the mass of a star. Instead, we must convert from the measured quantity, the luminosity function $\psi(M)$, the number of stars in a magnitude range $M \rightarrow M+d M$ (note that $M$ refers to magnitude while $m$ refers to

\footnotetext{
${ }^{1}$ There have been several attempts to determine the mass function of local stars (Miller \& Scalo 1979, Kroupa, Tout \& Gilmore 1993, Henry 1995). Local stars are predominantly Population I disk stars. A population II MF for low mass stars from globular cluster NGC 1261 can be found in Zoccali et al (1995). In our paper we focus instead on local field halo stars, which are not in globular clusters.
} 
mass). For the luminosity function (hereafter LF), we use units \#stars $/ \mathrm{pc}^{3} / M$.

To obtain a mass function for red dwarfs, we use the LF from the extensive parallax survey being carried out by the US Naval Observatory. Preliminary results were reported in Monet et al (1992) with a more complete update in Dahn et al (1995). They published an LF based on 114 stars with velocities tangential to the line of sight $V_{\tan } \geq 220 \mathrm{~km} / \mathrm{sec}$. These high velocity stars should all be halo stars (temporarily in the disk) since true disk stars have a local velocity dispersion of only $\sim 30 \mathrm{~km} / \mathrm{sec}$. Méra, Chabrier \& Schaeffer (1996) used this LF to derive an MF as discussed further below; see their Fig. 1. In this paper we present a more complete derivation of the mass function for red dwarfs, and a different interpretation. We take into account several potentially important effects not considered by Méra et al, such as unresolved binaries in the sample and the effects of different choices of metallicity. In particular, many of the 'stars' in the sample are likely to be unresolved binaries, and substantial numbers of binaries may drastically alter the results. The MF derived by Méra et al falls into the range of possibilities bracketed by the parameter range we consider. However, our interpretation is different. We emphasize a different slope of the MF near the hydrogen burning limit. (By "slope", we mean the $\log$ arithmic slope $\mathrm{d} \log \xi / \mathrm{d} \log m$.) In addition we use theoretical results from star formation to extrapolate to lower masses and limit the abundance of halo brown dwarfs. Because we are using parallax data, we can only discuss the local population of halo stars.

\section{Deriving the MF of Red Dwarfs}

As described above, we use the LF obtained from the parallax survey of Dahn et al (1995). To convert from the luminosity function $\psi\left(M_{V}\right)$ to the mass function $\xi(m)$, we use

$$
\xi(m)=\left|\frac{d M_{V}}{d m}\right| \psi\left(M_{V}\right) .
$$

Clearly we need a mass-luminosity relation. We use the models calculated by Alexander et al (1996). In Fig. 8 of their paper, one can see that the mass-luminosity relation is non-linear. The luminosity of stars drops fairly slowly with decreasing mass until an inflection point at $m \approx 0.4 M_{\odot}$. Then the mass-luminosity relation turns over, and the luminosity rapidly becomes dimmer as the mass decreases toward $m_{H}$. We fit between the calculated points of (Alexander et al. 1996) using cubic splines. Since our MF will depend on certain assumptions, we will always consider the most 'conservative' case, i.e., we generate the steepest possible MF as one goes to low mass, corresponding to the largest number of low mass red dwarfs and of brown dwarfs. 
We use the model of Alexander et al. with stellar metallicity $Z=3 \times 10^{-4}$. Nearly all stars in the sample have larger metallicities than this, as can be seen from the comparison of parallax data points with theory for various metallicities in Fig. 6 of Alexander et al. (1996). We use this value of $Z$ because it gives the most conservative result (the steepest MF towards low mass). One can see this effect in Fig. 1a for two different metallicities and can understand it as follows. High Z stars are dimmer than low Z stars of the same mass, while high Z stars are more massive than low Z stars of the same luminosity. For the range of luminosities we are considering, if we underestimate $\mathrm{Z}$, stars would be on a much steeper part of the $M_{V}(m)$ curve and many would have masses in a narrow mass range close to the hydrogen burning limit $m_{H}$. In addition, the factor $\left|d M_{V} / d m\right|$ of Eq. (11) becomes large for stars near $m_{H}$ (because the mass range for low $\mathrm{Z}$ stars of a given luminosity is so small). These two effects combine to cause the MF to steepen towards lower mass as we lower the metallicity. Thus, using $Z=3 \times 10^{-4}$ gives the steepest allowed MF and the largest number of brown dwarfs.

In addition, the reported LF can differ from the true LF because of potential errors and biases in the parallax data. Observers on average underestimate the luminosity of the stars because there are more distant stars than nearby stars (Lutz \& Kelker 1973). Also, due to random errors, there is a diffusion-like broadening of the LF (Stobie, Ishida \& Peacock 1989) so that the observed LF does not drop as fast as the true one as one goes to dim stars. Both systematic biases (known as Malmquist bias) will cause us to have two many small dim stars in our sample, so we will over-estimate the steepness of the MF as one goes to small mass. Again, our results are conservative.

The LF derived by Dahn et al. grows to a maximum value at $M_{V}=12$ and then drops off again as one goes to lower mass. [Hereafter, we use the words rising and falling to refer to the behavior of functions as one goes to smaller values of mass or of luminosity.] One might naively interpret this to mean that there cannot be many stars of low mass. However, this interpretation may not be correct because of the shape of the mass-luminosity curve (Kroupa, Tout \& Gilmore 1990); 5LF that reached a maximum and then turned over for all a decreasing luminosity function does not necessarily imply a decreasing MF.

A Complication: Binaries: A complication arises in the implementation of Eq. (1): some of the stars in the survey may actually be unresolved binaries. If so, the luminosity of the binary is due to light from both stars. If we mistakenly interpret the light to be only from a single star, we may overestimate the mass of the star. To deal with this potential problem, we make a variety of assumptions about unresolved binaries, and plot the corresponding mass functions in Fig. 1. Our models represent extremes which bracket 
the possible range of MFs due to unresolved binaries. We shall see that for all cases, we still conclude that brown dwarfs are an insignificant component of the mass of the halo.

In Model I we make the most common assumption about binary stars: the masses of the two stars are independent and yet are drawn from the same distribution; i.e., there is a single MF which determines the mass of each star. Note that we do allow the binary companion to have a mass below the hydrogen burning limit (we believe that there is no physical reason to cut off the distribution here). Thus our model (and its conclusions) is different from that of other authors (Kroupa, Tout \& Gilmore 1991, 1993; Piskunov and Malkov 1991) who do not consider brown dwarf companions. We here show that if the mass density in brown dwarfs is very small for the no binary case, then it is also very small for the case of model I. Assume that brown dwarfs are far more numerous than true stars. Then by our assumption that the masses of the two stars are drawn from the same MF, the secondary in any binary system is nearly always ${ }^{2}$ a brown dwarf 3 . The contribution of the brown dwarf to the total luminosity of the system is negligible and we can derive the mass of the primary from the luminosity of the system. This means that the MF derived using a single star mass-luminosity relation also describes the MF for binary stars. But, as we will show below, this single star MF implies that there are not large numbers of brown dwarfs, contradicting our assumption. Thus, if the mass density in brown dwarfs is very small for the no binary case, then it is also very small for the case of model I.

Although model I is the most plausible, it is difficult to directly calculate the actual mass function from the observed massfunction (although the reverse process is trivial). Thus we tried two other binary star models. Model II is plausible. Model III is extremely unlikely, and is designed to exaggerate the shift in slope of the MF. In both models, we analytically determined the MF which would produce the observed LF and which would satisfy the assumed binary composition. Both models steepen the MF towards low masses. [Note that the direction of this effect is in agreement with what was found by Kroupa, Tout, \&

\footnotetext{
${ }^{2}$ Since the above authors did not allow brown dwarf companions, this statement would not be true for their work; our model II more closely approximates their results.

${ }^{3}$ There has been an intensive search for brown dwarfs as binary companions. This search has only found two brown dwarfs despite examining hundreds of systems; thus brown dwarfs make up $<1 \%$ of the mass of disk stars (Rebolo, Zapatero Osorio \& Martin 1995; Nakajima et al. 1995). Because brown dwarfs cool as they age, one can only hope to optically detect young brown dwarfs. Thus, these optical searches are not sensitive to a halo population of brown dwarfs.
} 
Gilmore (1991, 1993), who studied the effects of unresolved binaries in other data sets (typically Pop. I)].

In model II, each "star" has a probability $f$ (the binary fraction) of being an unresolved binary. In every binary system, we take the mass of the secondary $m_{s}$ to be evenly distributed between zero and the mass of the primary $m_{p}$; i.e., the binary mass ratio $q=\frac{m_{s}}{m_{p}}$ has an equal chance of being anywhere in the interval [0,1]. For each bin $M_{V}$ in the observed LF histogram $\psi^{\prime}\left(M_{V}\right)$, a fraction $(1-f) \psi^{\prime}\left(M_{V}\right)$ of the LF is assumed to represent single stars whose absolute magnitude has been accurately determined. The remaining fraction $f \psi^{\prime}\left(M_{V}\right)$ represents all unresolved binaries and is divided into 100 parts $i$, each having a mass ratio $q_{i}=i / 100$, where $i$ is an integer between 0 and 100. For each part $i$ we find the two masses $m_{p, i}$ and $m_{s, i}=q_{i} m_{p, i}$ with visual magnitudes $M_{V p, i}$ and $M_{V s, i}$ such that the combined luminosity of the system is exactly what is observed. We then put these pieces together to obtain the luminosity function $\psi\left(M_{V}\right)$ corrected for unresolved binaries; the results from model II will be discussed shortly.

In model III, we assume that all of the stars are in binary systems, and that the two stars have the same luminosity. Thus the luminosity of the primary is half that of the system. Compared to the no-binary case, in model III the luminosity of each star is half as much, the magnitude is larger by an amount 0.75 , and the mass of each star is smaller. Also, there are twice as many stars because each system is a binary. Thus, according to model III, the "true" LF $\psi\left(M_{V}\right)$ can be derived from the observed LF $\psi^{\prime}\left(M_{V}\right)$ by: $\psi\left(M_{V}\right)=2 \times \psi^{\prime}\left(M_{V}-0.75\right)$. Although the shape of the LF is unchanged, this model generates a steeper MF for the same reasons a lower metallicity model generates a steeper MF: stars lie on a steeper part of the $M_{V}(m)$ curve so that their masses lie in the narrow mass range near the hydrogen burning limit, and the factor $\left|d M_{V} / d m\right|$ of Eq. (1) becomes large as discussed in section 2.

Our results for all the models are plotted in Fig. 1. The MF of red dwarfs has been obtained from the LF using Eq. (11) for four different cases. Both binary models II and III generate MFs that are steeper than if we ignored unresolved binaries. In the plots, the MF has been multiplied by $m^{2}$ so that one can most easily see how it approaches the hydrogen burning limit (as described in the next section). Our result is that, at the low mass end, the MF behaves as $\xi(m) \sim m^{-\alpha}$ with $\alpha \leq 2$ for all four cases we consider. In fact, only binary model III could have $\alpha=2$ while all other cases have $\alpha<2$.

For comparison, Méra et al (1996) found the MF only for the case of no binaries, and roughly estimated $\alpha \sim 2$. They obtained this number by fitting to the MF over a large mass range. However, we find that for this case of no binaries, the slope $\alpha$ is smaller near the hydrogen burning limit, as one can see from Fig. $1 \mathrm{a}$; it is this smaller slope at the lowest mass end of the red dwarf range that is relevant for extrapolating 
into the brown dwarf regime f $^{\text {B B }}$ Bew we also take advantage of recent results from star formation theory to guide our extrapolation, and this leads us to a different interpretation of the importance of the shape of the MF near the hydrogen burning limit. Also, our work has considered a wider range of possibilities for binaries with the aim of obtaining a careful estimate of the range of possibilities for $\alpha$. As seen in the next section, the fact that we find a slope which always satisfies $\alpha \leq 2$ has important consequences.

\section{Upper Limit on the Total Mass of Halo Brown Dwarfs}

Extrapolating the MF to Brown Dwarfs - Theoretical Input: Given our mass functions of halo red dwarfs, we will now extrapolate to the lower mass brown dwarfs. This extrapolation should be guided by theory. The work of Adams and Fatuzzo (1996) predicts the mass of a star based on the physical properties of the cloud core that forms that star. In their theory, stars determine their own masses through strong stellar winds and outflows. They predicted two extremes for the MF of stars forming in molecular clouds. At one extreme, the stellar mass is determined by one factor, e.g., the sound speed of the molecular cloud core. In this extreme, the mass function of stars would be a power law. At the other extreme, the mass of the star is determined by a large number of independent physical variables. Invoking the central limit theorem, Adams and Fatuzzo reasoned that $\log \xi(m)$ should be a Gaussian; i.e., the mass should be log-normal distributed 5 .

These two cases represent extreme limits. We expect the actual function to lie somewhere between the two. In short, a MF should not be concave up, only flat or concave down on a log-log plot. There are other current theories of star formation based on completely different physical reasoning, such as heirarchical fragmentation (Zinnecker 1984) and collisions between cloud cores (Price \& Podsiadlowski 1995). All of these theories predict mass functions that rise less quickly than power laws, and thus lie within the same range of possibilities as discussed above. All the possible MFs generated by our different models are consistent with a mixture of the two theoretical models over the red dwarf mass range, i.e., all are flat or concave down.

\footnotetext{
${ }^{4}$ Méra et al (1996) found $\alpha \sim 2.5$ for the data of Richer and Fahlman (1992); however these data had difficulty distinguishing least luminous stars from galaxies and disagree with the results of Hubble Space Telescope (Bahcall etal 1994, Graff and Freese 1996).

${ }^{5}$ To leading order, the MF of disk stars appears to be fit by a log-normal (Miller \& Scalo 1979).
} 
Upper limit on the total mass in Halo brown dwarfs: The total mass in brown dwarfs is

$$
m_{\mathrm{tot}}=\int_{0}^{m_{H}} m \xi(m) d m
$$

Since there are so few red dwarfs relative to the halo, the total mass of brown dwarfs can only be large if the MF is steeply rising as one goes to small masses. In the theory discussed above, any possible extrapolation of an MF lies between two possible extremes: power law or log-normal. Within these two bounds, there will be more brown dwarfs if we extrapolate with a power law. Hence we use $\xi \propto m^{-\alpha}$ to place an upper bound on the total mass of brown dwarfs in the halo. Note that we extrapolate the slope at the low mass end of the observed mass function.

If $\alpha<2$ then the integral in equation (2) converges and one finds the total mass in brown dwarfs to be of the same order of magnitude as the total mass in red dwarfs. If $\alpha \geq 2$, then the integral diverges. Since the mass density of brown dwarfs is not infinite, we mean by this divergence that the total mass density is dependent on a lower mass cutoff of the MF. If $\alpha=2$ then the integral diverges, but only logarithmically, i.e., each order of magnitude of mass range contains an equal total mass. Thus, even for a lower limit of $10^{-7} M_{\odot}$, the total mass in brown dwarfs $\sim 12$ times the mass in red dwarfs, or only $3 \%$ of the local halo mass. If $\alpha>2$, the integral diverges fast enough that there could be enough brown dwarfs to fill the halo.

As shown in Fig. 1, for all of our models, with varying metallicities and binary content, a power law extrapolation to low masses has a power of $\alpha \leq 2$. Thus brown dwarfs cannot contribute more than $\sim 3 \%$ of the local halo mass. Only our most extreme model (binary model III) can have $\alpha=2$; the more sensible models have $\alpha<2$. Eq. (2) with $\alpha<2$ implies that there cannot be many more brown dwarfs than red

dwarfs. Previously we estimated that red dwarfs contribute $\sim 0.3 \%$ of the halo (for a halo mass density of $\left.7 \times 10^{-3} M_{\odot} / p c^{3}\right)$. In summary, brown dwarfs cannot contribute more than $\sim 3 \%$ of the mass of the halo, and in realistic models the limit is an order of magnitude smaller.

\section{Discussion}

Red dwarfs are a tiny fraction, $0.3 \%$, of the mass of the galactic halo (Graff \& Freese 1996). In order for any appreciable fraction of the halo mass to consist of low mass star-like objects, there must be substantially more mass in brown dwarfs than in red dwarfs. We estimated the mass in brown dwarfs by extrapolating the mass function (MF) of red dwarfs below the hydrogen burning limit. Our extrapolation was based on current theories of star formation, which predict that the MF is either flat or concave down 
on a log-log plot (or in fig. 1), i.e., the MF rises no faster than a power-law. We made several assumptions about the metallicity and binary composition of the stars in the sample. We found that likely extrapolations imply that brown dwarfs make up less than $\sim 3 \%$ of the local mass density of the halo. For our most realistic models, the limit on the total mass in brown dwarfs is roughly the total mass in red dwarfs, $\sim 0.3 \%$ of the local mass density of the halo.

To repeat an earlier caveat: our limits on the brown dwarf density assume that the brown dwarfs in the halo come from the same stellar population as the red dwarfs. The possibility always remains that there are large numbers of brown dwarfs from an entirely different population of stars (Population III). However, theoretical work on star formation (Adams and Fatuzzo 1996) indicates that an earlier population of stars is likely to be skewed towards a predominance of high mass stars, not brown dwarfs. There may also be some yet undiscovered mechanism of star formation which allows the MF to rise faster than power-law. In this paper we have presented red dwarf mass functions and have shown that brown dwarfs which are members of known stellar populations are not present in the halo in great abundance.

We thank Santi Cassisi for sharing unpublished work with us. We also thank F. Adams, G. Basri, P. Kroupa and J. Bahcall for useful discussions. We acknowledge support from NSF PHY-9406745 and from the Univ. of Michigan Physics Dept. 


\section{REFERENCES}

Adams, F. C. \& Fatuzzo, M. 1996 ApJ, in press.

Alcock, C. et al. 1996, ApJ461 84.

Alexander, D. R., Brocato, E., Cassisi, S., Castelliani, V., Ciaco, F., \& Degl'Innocenti, S. 1996 submitted to A\&A.

Ansari, R. et al. 1996 submitted to A\&A.

Bahcall, J., Flynn, C., Gould, A. \& Kirhakos, S. 1994, ApJ, 435, L51.

Boeshaar, P. C., Tyson, J. A. \& Bernstein, G. M., 1994, BAAS, 185, \#22.02.

Burrows, A., Hubbard, W. B., Saumon, D. \& Lunine, J. I., 1993, ApJ, 406, 158.

Burrows, A. \& Liebert, J. 1993, RMP, 65, 301.

Dahn, C. C., Liebert, J, Harris, H. C. \& Guetter, H. H. 1995, in Proceedings of the ESO workshop "The bottom of the Main Sequence and Beyond" ed. C.G.Tinney (Springer-Verlag, Heidelberg) 239.

Graff, D. S. \& Freese, K. 1996 ApJ, 456, L49.

Henry, T. 1995, in Proceedings of the ESO workshop "The bottom of the Main Sequence and Beyond" ed. C.G.Tinney (Springer-Verlag, Heidelberg) 79.

Kroupa, P., Tout, C. A., Gilmore, G. 1990 MNRAS, 244, 76.

Kroupa, P., Tout, C. A., Gilmore, G. 1991 MNRAS, 251, 293.

Kroupa, P., Tout, C. A., Gilmore, G. 1993 MNRAS, 262, 545.

Lutz, T. E., \& Kelker, D. H. 1973, PASP, 85, 573.

Miller, G. E. \& Scalo, J. M. 1979 ApJS, 41, 513.

Méra, D., Chabrier, G. \& Schaeffer, R. 1996 Europhys. Lett., 33, 327.

Monet, D. et al. 1992 AJ, 103, 639. 
Nakajima, T., Oppenheimer, B. R., Kulkarni, S. R., Golimowski, D. A., Matthews, K. \& Durance, S. T. 1995 Nature, 378, 463.

Piskunov \& Malkov, 1991 Astronomy and Astrophysics, 247, 87.

Price, N. M. \& Podsialdlowski, P. 1995 MNRAS273, 1041.

Rebolo, R., Zapatero Osorio, M. R. \& Martin, E. L. 1995 Nature, 377, 129.

Richer, H.B. and Fahlman, G.G. 1992 Nature, 358, 383.

Stobie, R. S., Ishida, K. \& Peacock, J. A. 1989, MNRAS238, 709.

Zinnecker, H. 1984 MNRAS210, 43.

Zoccali, M., Piotto, G., Zaggia, S. R. \& Capaccioli, M. 1995, in Proceedings of the ESO workshop "The bottom of the Main Sequence and Beyond" ed. C.G.Tinney (Springer-Verlag, Heidelberg) 261.

This manuscript was prepared with the AAS IATEX macros v4.0. 


\section{F I G U R E C A P T I O N S}

Figure 1: The mass function of red dwarf halo stars. Each of the four models is derived from the LF of Dahn et al (1995) but assumes different metallicity and binary content. In all three panels, crosses without errorbars illustrate the mass function derived for stars with metallicity $\mathrm{Z}=3 \times 10^{-3}$ and no binary companions. Error bars are due to Poisson errors in the LF. The other model presented in panel (a) has $\mathrm{Z}=6 \times 10^{-3}$ (no binaries) for comparison. Lower metallicity leads to a MF with more low mass stars. Panels (b) and (c) show binary models II and III for $Z=3 \times 10^{-3}$ as described in the text and can be compared with the no binary model (crosses) of the same metallicity. For binary models we plot the total MF (primaries and secondaries). Panel (b) shows model II for two different binary fractions, $f=\{0.5,1.0\}$; $f=0.5$ is closer to the no-binary model (crosses). Binary model II has a somewhat steeper MF than the no binary case. Binary model III has been designed to exaggerate the number of low mass stars compared to high mass ones and is unrealistic.

We have multiplied the vertical axis by $m^{2}$ to emphasize that all MFs converge at low mass (see eq (2) and subsequent text). A MF which is decreasing to the left will converge; one that is increasing will diverge. One that is flat will diverge, but only logarithmically. Even for the extreme case of binary model III, the MF is flat or decreasing and the total mass in brown dwarfs could be at most $\sim 3 \%$. For more likely models I and II, the limit on the total mass in brown dwarfs is very roughly the total mass in red dwarfs, $\sim 0.3 \%$ of the local mass density of the halo. 


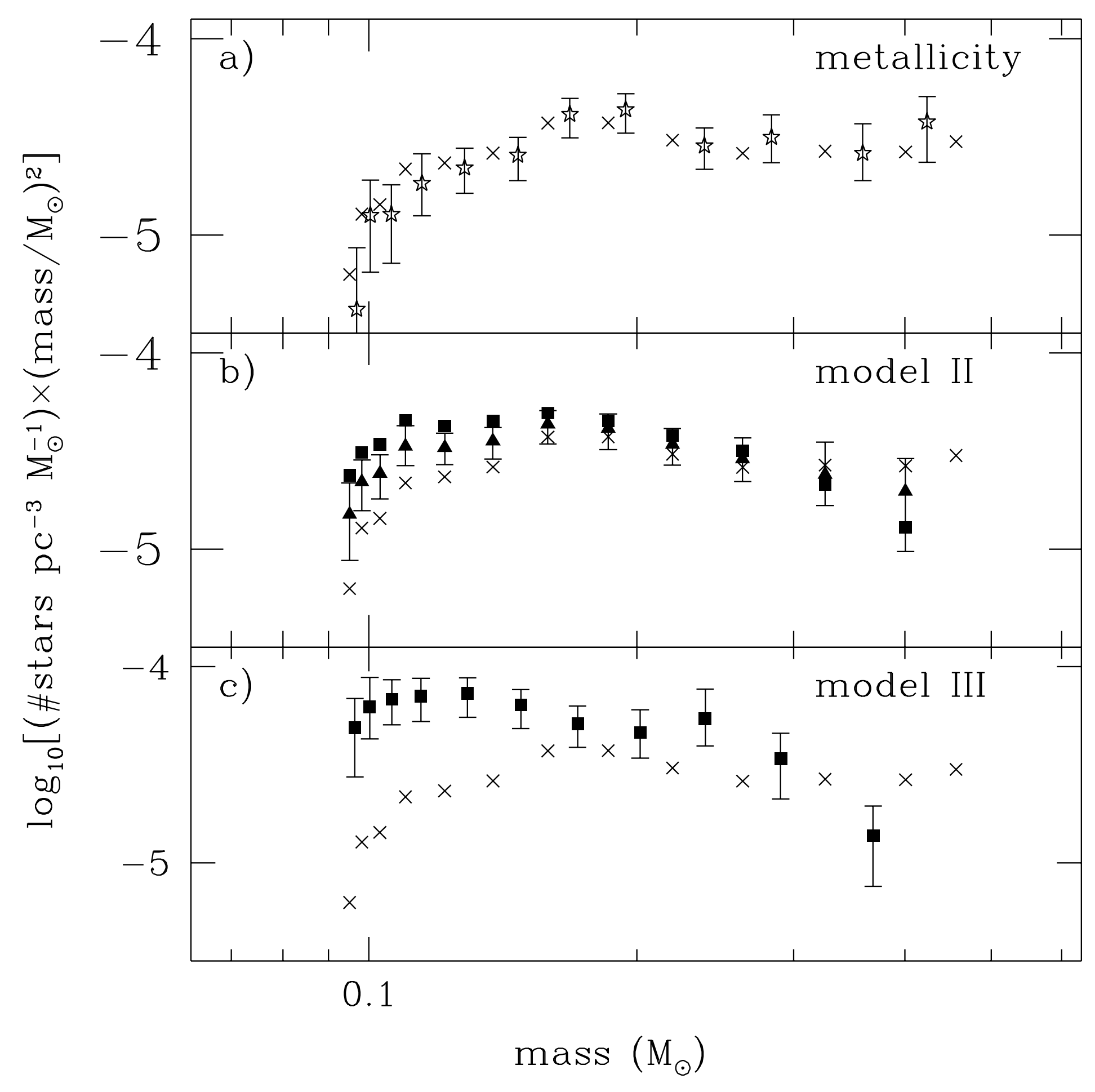

